\title{
Diagnóstico diferencial de massas abdominais na infância: revisão de literatura
}

\author{
Abdominal mass differential diagnosis in childhood - literature review \\ Nathalye Emanuelle Souza ${ }^{3}$, Letícia do Nascimento Botrel${ }^{1}$, Kleiton Santos Neves².
}

\begin{abstract}
Resumo
O objetivo desse estudo é discutir as características clínicas e laboratoriais das massas abdominais mais freqüentes na infância, contribuindo para o diagnóstico precoce destas doenças. Diversos estudos comprovam que a maioria das massas abdominais é causada por doenças benignas. Entretanto, neoplasias malignas da infância se manifestam de semelhante modo, como tumor abdominal. Conclusão: Através da análise dos dados clínicos e laboratoriais do paciente com tumor abdominal, o pediatra pode traçar hipóteses diagnósticas e encaminhar precocemente a criança para centros especializados em caso de tumores malignos. Palavras-chave: Infância. Massas abdominais. Tumor abdominal. Diagnóstico diferencial.
\end{abstract}

Como citar esse artigo. Souza NE, Botrel LN, Neves KS. Diagnóstico diferencial de massas abdominais na infância: revisão de literatura. Revista de Saúde. 2015 Jul./Dez.; 06 (2): 11-14.

\begin{abstract}
The objective of this study is to discuss the clinical and laboratory characteristics of the most common abdominal masses in children, contributing to early diagnosis of these diseases. Several studies show that most abdominal masses is caused by benign diseases. However, malignancies of childhood manifest themselves similarly as abdominal tumor. Conclusion: The analysis of the clinical and laboratory data of the patient with abdominal tumor, the pediatrician can trace diagnostic hypotheses and early refer the child to specialized centers in case of malignant tumors. Keywords: Childhood. Abdominal masses. Abdominal tumor. Differential diagnosis.
\end{abstract}

\section{Introdução}

As massas abdominais na infância apresentam diversas etiologias, em geral são doenças de curso benigno, tais como hidronefrose, rim policístico, malformação intestinal ou cistos. Diversos estudos comprovam que a maioria das massas abdominais é causada por doenças benignas. ${ }^{3,4}$ Entretanto, neoplasias malignas da infância se manifestam de semelhante modo, como tumor abdominal se fazendo necessário seu rastreio precoce, afim de preservar a vida da criança, levando ao aumento da mortalidade dessa faixa etária. ${ }^{78}$ Segundo a literatura, tumores malignos nesta faixa etária são raros, contudo, elucidar o raciocínio diagnóstico de forma precoce é extremamente imprescindível para garantir possibilidades de cura para o paciente. ${ }^{1,2}$ Diante disso, presente estudo tem por objetivo discutir as principais características das massas abdominais mais frequentes na infância, utilizando-se de estratégias para estabelecer diagnósticos diferenciais de tumores abdominais.

\section{Materiais e Métodos}

Revisão bibliográfica, através do banco de dados Scielo, PubMed, MedLine e LILACS, utilizando os termos: tumores abdominais, diagnóstico diferencial na infância, massas abdominais, câncer com massa abdominal, em inglês, português e espanhol, no período de 1995 a 2015. Foram selecionados artigos científicos que relataram estudos de caso, relato de caso, revisões de literatura e estudos multicêntricos sobre massas abdominais na infância.

\section{Resultados}

Cypriano et al., ${ }^{3}$ em sua revisão de literatura, discute as características clínicas e laboratoriais dos tumores malignos abdominais mais freqüentes na infância, contribuindo para o diagnóstico precoce dessas doenças, sendo essas características de suma importância para o prognóstico dos pacientes.

Neves et al. ${ }^{14}$ descrevem a experiência com crianças tratadas de tumores carcinoides e investigam a freqüência morfológica, enquanto Saito et al. ${ }^{8}$ abordam

1. Universidade Severino Sombra, Pró-reitoria de Ciências Médicas, Discente do Curso de Medicina.

2. Universidade Severino Sombra, Pró-reitoria de Ciências Médicas, Docente do Curso de Medicina.

3. Universidade Severino Sombra, Pró-reitoria de Ciências Médicas, Discente do Curso de Medicina, Bolsista PIBIC/USS. 
os principais tumores hepáticos na infância, nos levando a compreender que tanto tumores malignos quanto benignos devem ser investigados na presença de massa abdominal.

Oliveira et al. ${ }^{4}$ relatam a experiência do Hospital das Clínicas da UFMG no tratamento do linfoma de Hodgkin na infância, através de série de 31 casos da doença.

A descrição de um caso de tricofagia sem tricotilomania, através de relato de caso de Menra et al., ${ }^{5}$ nos leva a pensar que a massa abdominal talvez seja conseqüência de um transtorno psiquiátrico (tricotilomania).

O trabalho de Cartum et al. ${ }^{9}$ é, indubitavelmente, de grande impacto no conhecimento de diagnóstico diferenciais de massas abdominais em crianças, pois aborda as características do Nnuroblastoma, um dos tumores mais prevalentes.

Através do estudo de Schettini et al., ${ }^{6}$ podemos entender como diagnosticar enterite necrosante em recém-nascidos, um dos diagnósticos diferenciais mais prováveis de massa abdominal nesta faixa etária.

Raramente o pediatra, abordando uma recémnascida, pensará em cisto de ovário, sendo, portanto, o estudo de Souza et al. ${ }^{23}$ importantíssimo para o entendimento dessa patologia.

\section{Discussão}

\section{Identificação e História Clínica}

Quando o médico estiver deparado com um caso de massa abdominal em pacientes infanto-juvenis, a anamnese minuciosa é essencial para o diagnóstico. Dados como idade, sexo e intercorrências no parto são de extrema relevância. A idade da criança correlacionase diretamente com a natureza da tumoração. ${ }^{2,3}$ No período neonatal, tumores malignos são raros, tendo predomínio às doenças renais: hidronefrose, cistos renais e nefroma mesoblástico ${ }^{3}$, mas não se pode esquecer das malformações intestinais, como cisto mesentérico. ${ }^{11}$

$\mathrm{O}$ tumor maligno mais freqüente em recémnascidos é o neuroblastoma, ${ }^{18}$ enquanto o tumor maligno mais comum do trato geniturinário na infância é o tumor de Wilms. Sua incidência é estimada em 350 novos casos por ano nos EUA, ${ }^{21}$ por vezes diagnosticado intraútero, através da ultrassonografia; contudo, na maioria das crianças acima de um ano, o prognóstico é desfavorável, tendo o tumor possivelmente se disseminado. ${ }^{9}$

Hepatoblastoma, leucemias e linfomas são encontrados em crianças com dois a dez anos de

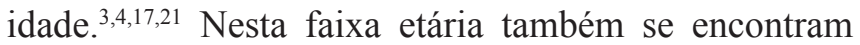
os tumores benignos, como os hemangiomas, os hamartomas hepáticos, ${ }^{7}$ algumas infecções que cursam com hepatoesplenomegalia, bolo de áscaris e fecaloma, ${ }^{24}$ ou, até mesmo, tricofagia em pacientes adolescentes, com desordens psíquicas. ${ }^{2,5}$

Em relação ao sexo, o cisto de ovário, apesar da raridade, é a causa mais freqüente de massa abdominal cística em feto ou recém-nascida. Anomalias do ovário e genitais correspondem a $20 \%$ de todas as massas abdominais da recém-nascida ( $\mathrm{RN})$, ultrapassadas em número pelas massas originadas no trato urinário, que representam $55 \%$ do total, aproximadamente. ${ }^{23}$

Uma anamnese detalhada, que explora minuciosamente a duração e as características dos sintomas, auxilia na elucidação do diagnóstico. A avaliação da presença de dor (se aguda ou crônica), presença de febre, perda de peso, cansaço ou dor óssea associadas são de extrema importância. Indagar sobre a constipação é de grande valia, visto que o fecaloma é a massa abdominal mais comum e muitas vezes detectada pelas próprias mães, ao palparem o abdome das crianças, o qual é fino e delgado. ${ }^{24}$ Caso a suspeita seja de um tumor, os sintomas desencadeados podem ser devido: ao volume cístico, à compressão de um segmento intestinal vizinho ou processos inflamatórios ao nível de mucosa. ${ }^{24}$

Através da História Patológica Pregressa e História Social, saberemos que, se houve contato com alguma doença infecciosa, essa pode predispor à formação de cistos, abscessos e adenomegalias. O hepatoblastoma fetal está relacionado com o baixo peso ou prematuridade, ${ }^{17}$ enquanto os adenomas hepatocelulares e hiperplasia nodular focal ${ }^{8}$ correlacionam-se ao uso de anticoncepcionais orais.

A História Familiar com ocorrência de neoplasias alerta-nos sobre a possibilidade de tumoração de origem neoplásica nestas crianças.

Todas estas, são situações que levarão pacientes e responsáveis ao consultório médico, em busca de um diagnóstico que esclareça o surgimento dessas massas.

Exame Físico

À inspeção, o abdome pode apresentar-se distendido, abaulado, herniado, com ascite ou circulação colateral. Quando palpá-lo, avalie cuidadosamente quaisquer sinais de obstrução intestinal, irritação peritoneal ou retenção urinária. A localização da massa abdominal (quadrante abdominal, intra ou retroperitoneal) deve ser analisada quanto à consistência, aderência, dor, mobilidade com a respiração e delimitações. $^{2}$

Na suspeita de doença abdominal é recomendado realizar o toque retal, como também o exame da genitália, seguida de pesquisa de hímen imperfurado e palpação de testículos.

Presença de puberdade precoce ocorre, geralmente, no carcinoma ou adenoma de suprarrenal e nos tumores de ovário (cabe ressaltar que apresentam 
pequeno volume, portanto só podem ser palpados pelo ginecologista ${ }^{24}$ e fígado.

Massa abdominal assintomática sugere cisto de mesentério, somente se não houver alterações significantes no hemograma, ${ }^{11}$ podendo também ser hepatoblastoma tipo fetal, se o paciente em questão for recém-nato. ${ }^{17}$

Exame laboratorial e de Imagem

Em diversas ocasiões, cabe ao médico pedir exames de rotina como Hemograma e Radiografias. O hemograma pode estar alterado em pacientes com leucemias e linfomas, através da presença de anemia, leucopenia ou plaquetopenia. ${ }^{3}$ Há um aumento expressivo da enzima desidrogenase lática (DHL) no linfoma não Hodgkin e leucemias.

Alfafetoproteína (AFP) geralmente encontrase elevada em pacientes com hepatoblastoma (em $90 \%$ dos casos, ${ }^{17}$ contudo baixos níveis de AFP levam ao pior prognóstico da doença), e tumores de células germinativas, assim como a gonadotrofina coriônica. Aumento de cortisol e hormônios sexuais nos tumores de suprarrenal. ${ }^{10}$

A radiografia (RX) de abdome pode revelar calcificações sugestivas de: cisto de mesentério ${ }^{11}$ (mesmo sendo incomuns e raramente sintomáticos, tem que estar na hipótese diagnóstica, visto a necessidade de intervenção cirúrgica), Neuroblastoma e tumor de células germinativas e formações ósseas sugestivas de teratoma.

A ultrassonografia (US) é um excelente método, de fácil acesso, custo baixo, não invasivo e indolor para a avaliação dos tumores abdominais, localizando o local da lesão e sua consistência, visto que a solidez se encontra na maioria das lesões malignas.

A tomografia computadorizada (TC) ou ressonância nuclear magnética (RNM), freqüentemente, complementam a ultrassonografia, fornecendo informações mais detalhadas sobre delimitações, além de características dos tumores. ${ }^{2}$

\section{Considerações finais}

Os tumores abdominais geralmente se apresentam como massa abdominal assintomática, muitas vezes descobertas em consultas de rotina ou pelos próprios pais. Dessa forma, a presença de massa abdominal exige uma maior atenção dos médicos para um diagnóstico mais precoce, através de um raciocínio bem elaborado e organizado da literatura médica atual, sabendo desde a prevalência até os principais achados laboratoriais e de imagem, levando a um maior sucesso quanto a real possibilidade de cura.

\section{Financiamento}

Agradecemos à Universidade Severino Sombra pela Bolsa PIBIC/USS.

\section{Conflitos de interesse}

Os autores declaram não haver conflitos de interesse.

\section{Referências}

1 Anveden-Hertzberg L; Gauderes MWL Abdominal Masses. In: Kliegman, Practical Strategies in Pediatric Diagnosis and Therapy, $1^{\mathrm{a}}$ edição. W.B. Saunders Company: Philadelphia, PA, 1996, pp 387-399.

2 Caran EMM; Luisi FAV; Cypriano M. Tumores abdominais malignos mais frequentes na infância: diagnóstico diferencial. Pediatria Moderna 2009; 45 (2): $60-64$

3 Cypriano M; Luisi FAV; Caran EMM. Tumores abdominais malignos mais frequentes na infância: diagnóstico diferencial. Pediatria Moderna 2013; 49 (3): 117 à 122

4 Oliveira BM, Viana MB, Cunha KCCMS. Linfoma de Hodgkin na infância: experiência de 16 anos em uma única instituição. J Pediatria; Rio de Janeiro;2000; 76(4):281-6.

5 Mehra A; Avatshi A; Gupta V et al. Trichophagia along with trichobezoar in the absence of trichotillomania, J Neurosci Rural Pract. 2014; 5(Suppl 1): S55-S57.

6 Schettini ST; Miyoshi MH. Moreira Jr,Enterocolite necrosante neonatal; http://www.moreirajr.com.br/revistas.asp? fase $=$ r003\&id materia $=832$ Acessado dia 15 de fevereiro de 2015 às $11 \mathrm{~h} 30$.

7 Esteves E; Goraib JA; Martins JL. Hamartoma mesenquimal hepático no recém-nascido; J Pediatria, Rio de Janeiro, 1997; 73(5):345-8.

8 Saito JT; Neto UF. Tumores hepáticos benignos. The Electronic Journal of Pediatric; Marco 2004, Volume 7; http://e-gastroped.com.br/mar04/tumores hepaticos.htm Acessado dia 05 de fevereiro de 2015 Às 10h27.

9 Cartum J, Neuroblastoma: o enigmático tumor da infância; Pediatria Moderna, Ago 2012 ;48 (8): 296 à 301

10 Silva MT; Filho AGO; Miranda ML. Pielonefrite xantogranulomatosa na infância: relato de casos. http:/www.moreirajr.com.br/revistas. asp? fase $=$ r003\&id materia $=2769$ Acessado dia 10 de fevereiro de 2015 às $10 \mathrm{~h} 33$.

11 Yasojima EY; Cruz MMM; Valente TON. Cisto de mesentério: Relatos de casos. Rev. Col. Bras. Cir., Rio de Janeiro Maio/Junho 2002; 29 (3):177178

12 Rodrigues KE; Camargo B. Diagnóstico precoce do câncer infantil: Responsabilidade de todos. Rev. Assoc. Med. Bras. 2003; 49(1): 29-34.

13 Poeira R ; Castanheira MC; Couto,HS; Fernandes,.; Botelho,M.Teratoma sacrococcígeo: do diagnóstico à cirurgia.Acta Pediátrica Portuguesa. 2010; $41(2): 101-103$

14 Neves GR; Chapchap P; Sredni ST. Childhood carcinoid tumors Description of a case series in a Brazilian cancer center. Sao Paulo Med J. 2006 Jan 5;124(1):21-5.

15 Çakan M; Koç A; Cerit K et al. A Case of Acute Myeloid Leukemia (FAB M2) with Inversion 16 Who Presented with Pelvic Myeloid Sarcoma: Case Report. Hindawi Publishing Corporation; Case Reports in Pediatrics2014; 2014:1-4

16 Nag D; Nandi A; Kumar P et al. Clear cell sarcoma of the kidney: A caseReport. [Disponível em: http://www.cancerjournal.net. Acesso em 05 de fevereiro de 2015; IP: 187.126.88.186] 
17 Spyridakis I; Kepertis C; Lampropoulos V et al. Embryonal/Fetal Subtype Hepatoblastoma: A Case Report. Journal of Clinical and Diagnostic Research.2014, Vol-8(9): ND01-ND02.

18 NELSON, Tratado De Pediatria, 19 ed, Elsevier, Rio de Janeiro, 2014.

19 Boechat PR; Nogueira ARR; Goraib JA. Linfangioma abdominal na criança: apresentação de oito casos. Jornal de Pediatria 1996;72(5): 335-340

20 Álvarez AM; Lamarre R. Estado del arte: Tumores de cresta neural, Neuroblastoma

fisiopatología, diagnóstico y tratamiento; Revista Med 20 (1): 38-49, 2012.

21 Dabus GC; Pereira IMR. Tumor de Wilms extra-renal: Relato de caso. Riol. Bras, 2004; 37 (4): 299-301.

22 Yandza T; Valayer J. Benign tumors of the liver in children: analysis of a series of 20 cases. J Pediatr Surg, 1986, v. 21, p. 419-423.

23 Souza JCK; Souza RFM. Cisto de ovário no feto e recém-nascida. Mom. \& Perspec. Saúde 2003;16(1):41-46

24 Herlt M, Pediatria: Diagnóstico diferencial; Cultura Médica, Rio de Janeiro, 1980, p 518-521. 